\title{
Celdas en cuña para refractometría: criterios de selección de materiales y parámetros
}

\author{
Wedge cells for refractometry: materials and parameters selection criteria
}

\author{
P. M. E. Vázquez ${ }^{* 1}$, E. V. Oreglia*2, L. Ciocci Brazzano*†3, F. E. Veiras ${ }^{* \dagger 4}$, C. L. Matteo* ${ }^{* 5}$ and P. A. Sorichetti*6 \\ *Universidad de Buenos Aires, Facultad de Ingeniería, \\ Grupo de Láser, Óptica de Materiales y Aplicaciones Electromagnéticas (GLOMAE) \\ Paseo Colón 850, C1063ACV, Buenos Aires, Argentina \\ 1 pvazquez@fi.uba.ar \\ 2 eoreglialfi.uba.ar \\ 3 bciocciefi.uba.ar \\ ${ }^{4}$ fveirasefi.uba.ar \\ ${ }^{5}$ cmatteodfi.uba.ar \\ ${ }^{6}$ psorichefi.uba.ar \\ ${ }^{\dagger}$ Consejo Nacional de Investigaciones Científicas y Técnicas (CONICET) \\ Godoy Cruz 2290, C1425FQB, Buenos Aires, Argentina
}

Recibido: 23/08/19; Aceptado: 02/01/20

\begin{abstract}
Resumen-Este trabajo presenta criterios de selección de materiales y parámetros de celdas en cuña para refractometría en el rango visible. Estas celdas consisten en dos prismas de ángulo recto enfrentados, que definen una cavidad con forma de cuña entre ellas, en la que se coloca la muestra cuyo índice de refracción se desea medir. Éste se determina a partir de la desviación de un haz luminoso que atraviesa la celda. Este modelo para fines de ingeniería tiene en cuenta la geometría de la celda y los índices de refracción del material de la celda y de la muestra. A partir de allí realizamos el cálculo numérico y trazado de rayos correspondiente al sistema óptico. También proponemos ajustes polinómicos para simplificar el tratamiento numérico, como complemento de las ecuaciones completas. Estos polinomios dan el índice de refracción de la muestra como una función explícita del ángulo de deflexión. Se incluye además el análisis de los errores de la aproximación.
\end{abstract}

Palabras clave: instrumentación óptica, refractometría, metrología.

Abstract- This work presents the materials and parameters selection criteria of wedge cells for refractometry in the visible range. These cells consist of two right angle prisms facing each other, defining a wedge-shaped cavity where the sample is placed. The refractive index of the sample is determined from the deviation of a light beam that traverses the cell. This model for engineering purposes includes the cell geometry and the refractive indexes of the cell material and the sample. We perform the numerical calculation and ray tracing of the optical system. We also propose polinomial fitting functions in order to simplify the numerical treatment, and as a complement to the complete set of equations. These polynomials give the refractive index of the sample as an explicit function of the deviation angle. An analysis of the approximation errors is also given.

Keywords: optical instrumentation; refractometry; metrology.

\section{INTRODUCCIÓN}

La refractometría en el rango visible resulta de sumo interés, tanto por sus aplicaciones tecnológicas como en investigación básica. Este método óptico de caracterización presenta ciertas ventajas en comparación con otras técnicas: no requiere contacto directo con la muestra, no implica alteraciones de la misma y el costo de su implementación no es elevado. Las condiciones de ensayo son seguras (aspecto de gran importancia en mediciones de líquidos inflamables o peligrosos) [1] y pueden variarse dentro de un amplio rango. Estas características hacen atractiva esta técnica para su aplicación en control de procesos industriales [2], [3].

Numerosas implementaciones de la técnica de refractrometría se encuentran en la literatura. Las disposiciones más importantes son las basadas en la determinación del ángulo límite para reflexión total interna (métodos de Abbe y de Pulfrich) [4], [5] o bien en la deflexión de un haz [6]-[8]. En la actualidad, este último grupo se basa en la implementación originalmente descripta por Hughes [9]. En ésta, se utiliza una celda en cuña, típicamente construida con dos prismas de ángulo recto enfrentados, cuyas caras laterales se encuentran en planos verticales. La forma externa de la celda es la de un prisma rectangular, definiendo una cavidad con forma de $\mathrm{V}$ en su interior, donde se coloca la muestra cuyo índice de refracción se desea medir (Fig. 1). Se hace incidir un haz de luz en un plano horizontal, ajustando el ángulo de incidencia en la cara frontal de forma tal que salga normal a la cara posterior de la celda. En esas condiciones, conocido el ángulo de incidencia, por ejemplo con un montaje goniométrico, se puede determinar el índice de refracción de la muestra bajo análisis.

El objetivo de este trabajo es presentar los criterios de selección de materiales y parámetros de celdas en cuña para la medición del índice de refracción en el rango visible. Los parámetros de diseño del sistema óptico son los ángulos e índices de refracción de los prismas a utilizar para su construcción y el rango de índices de refracción de las muestras que se buscan caracterizar. Además es necesario seleccionar las dimensiones de la celda teniendo en cuenta 


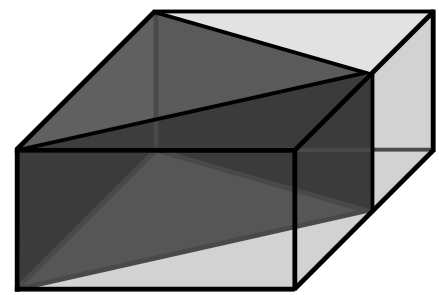

Figura 1: Esquema de la celda en cuña. Consta de dos prismas de ángulo recto enfrentados (gris claro) que definen una cavidad (gris oscuro), donde se introduce la muestra con índice de refracción a medir.

las limitaciones de espacio y el volumen de muestra disponible.

\section{DESARROLlo TEÓRICO}

En esta sección desarrollamos el procedimiento de cálculo para el diseño de celdas en cuña, en particular, presentamos las ecuaciones correspondientes y sus soluciones. Un corte transversal de la celda se muestra en la Fig. 2. En la misma se indican los elementos del diseño: los índices de refracción de los prismas $\left(n_{2} \mathrm{y} n_{4}\right)$, el índice de la muestra $\left(n_{3}\right)$, los ángulos de los prismas $\left(\omega_{1}, \omega_{2}\right.$ y $\left.\omega_{3}\right)$, y el ángulo de incidencia $\left(i_{1}\right)$ sobre la cara frontal, a la entrada de la celda. Además supondremos que la celda está inmersa en un medio de índice de refracción $\left(n_{1}\right)$, usualmente, aire.

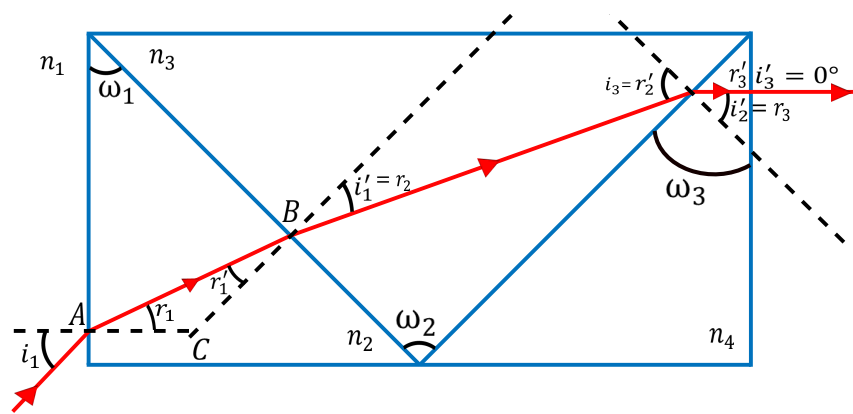

Figura 2: Corte transversal de la celda. Se indica el trazado de rayos, los índices de refracción y ángulos de incidencia y refracción en las interfaces.

Llevamos a cabo los cálculos considerando que en el sistema óptico descripto existen cuatro interfaces donde tiene lugar la refracción del haz luminoso:

1. Entre el medio en el que está inmersa la celda, por ejemplo aire, y el primer prisma que la conforma.

2. Entre el primer prisma y la cavidad con forma de cuña que contiene la muestra.

3. Entre la cavidad con la muestra y el último prisma de la celda.

4. Entre el último prisma y el medio en el que está sumergida la celda.

\section{II-A. Refracción en interfaces}

Para el cálculo, nos basamos en las Leyes de la refracción de Snell [10]:

$$
n_{1} \sin i_{1}=n_{2} \sin r_{1}
$$

donde $n_{1}$ es el índice de refracción del medio en el que está inmersa la celda (en general, aire); y $r_{1}$, el ángulo de refracción luego de la primer interfaz. Ambos ángulos, $i_{1}$ y $r_{1}$, son medidos con respecto a la normal al plano en el punto de incidencia $A$, sobre la primer interfaz. En el caso de la segunda interfaz, entre el primer prisma y la cavidad con forma de cuña:

$$
n_{2} \sin r_{1}^{\prime}=n_{3} \sin i_{1}^{\prime}
$$

donde $r_{1}^{\prime}$ e $i_{1}^{\prime}$ son los ángulos de incidencia y refracción en la segunda interfaz.

De la relación de ángulos en el triángulo $\mathrm{ABC}$ (Fig. 2) en el primer prisma, se sabe que el ángulo refringente, $\omega_{1}$, entre los dos planos no paralelos [10], es

$$
\omega_{1}=r_{1}+r_{1}^{\prime}
$$

Análogamente, para el caso de los dos prismas restantes:

$$
\begin{aligned}
& \omega_{2}=r_{2}+r_{2}^{\prime} \\
& \omega_{3}=r_{3}+r_{3}^{\prime}
\end{aligned}
$$

Aplicamos la Ley de Snell al caso de las interfaces restantes:

$$
\begin{aligned}
& n_{3} \sin r_{2}^{\prime}=n_{4} \sin i_{2}^{\prime} \\
& n_{4} \sin r_{3}^{\prime}=n_{5} \sin i_{3}^{\prime}
\end{aligned}
$$

Entonces, a partir de los valores de los parámetros de diseño, para un dado ángulo de salida $i_{3}^{\prime} \mathrm{y}$ de las ecuaciones 1 a 7 , se puede calcular el ángulo de incidencia $i_{1}$ a la entrada de la celda. Este está relacionado de manera unívoca con el índice de refracción $n_{3}$ de la muestra a caracterizar. Dado que en este trabajo imponemos que el haz de salida sea normal a la cara de la celda, la ecuación 7 se satisface idénticamente $\left(i_{3}^{\prime}=0^{\circ}\right)$.

\section{II-B. Trazado de rayos}

Realizamos de manera computacional la marcha de rayos dentro de la celda y la prolongación a su exterior (Fig. 3), con el objetivo de poder determinar las limitaciones espaciales que existen, y que acotan el área en la que se puede trabajar.

El sistema de coordenadas considerado tiene su origen en el vértice del prisma donde incide la luz. Sus ejes coinciden con los lados del primer prisma (Fig. 3).

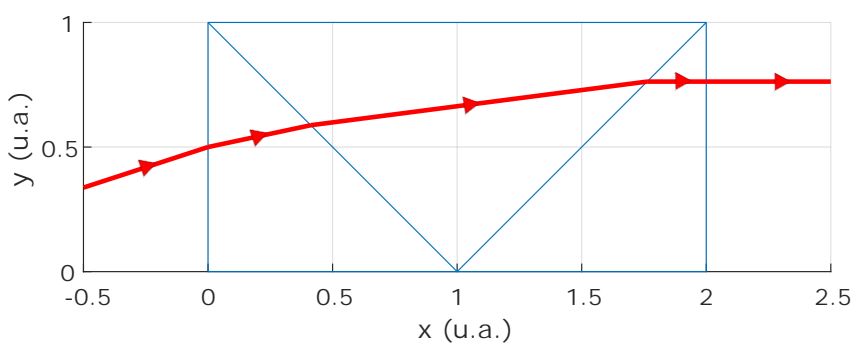

Figura 3: Trazado de rayos computacional efectuado con las ecuaciones planteadas en el texto. Las unidades son arbitrarias, normalizadas a la longitud del lado de los prismas seleccionados. En este caso particular, $\omega_{1}=\omega_{3}=45^{\circ}$; $\omega_{2}=90^{\circ} ; n_{1}=1$ (aire); $n_{2}=n_{4}=1,49 ;$ y $n_{3}=1,33$ (agua destilada), por ende, $i_{1}=18^{\circ}$. 
A través del esquema espacial, se puede determinar la posición del punto de incidencia del haz de luz, de manera tal que su camino se mantenga dentro de la celda luego de cada refracción. Esto, a su vez, sirve para determinar la posición del punto de salida del haz (en $x=2$, Fig. 3). Teniendo en cuenta los aspectos previamente mencionados, se pueden determinar las dimensiones del sistema de detección.

El diseño del sistema óptico se realiza de forma que el haz de luz salga normal a la cara posterior de la celda [9]. Por consiguiente, para determinar el índice de refracción de la muestra, sólo hay que medir el ángulo de incidencia.

\section{RESULTADOS}

En esta sección se presentan los resultados de la metodología de cálculo expuesta anteriormente. En primer lugar, se presenta el gráfico del ángulo de incidencia en la cara frontal de la celda $\left(i_{1}\right)$ en función del índice de refracción del material de la celda (caso $n_{c}=n_{2}=n_{4}$, de la notación utilizada en las secciones anteriores), para cuatro índices de refracción distintos de la muestra, $n_{s}=n_{3}$ (Fig. 4). Los valores de $n_{s}$ elegidos cubren el rango usual de sustancias de interés, desde agua pura hasta soluciones de tierras raras.
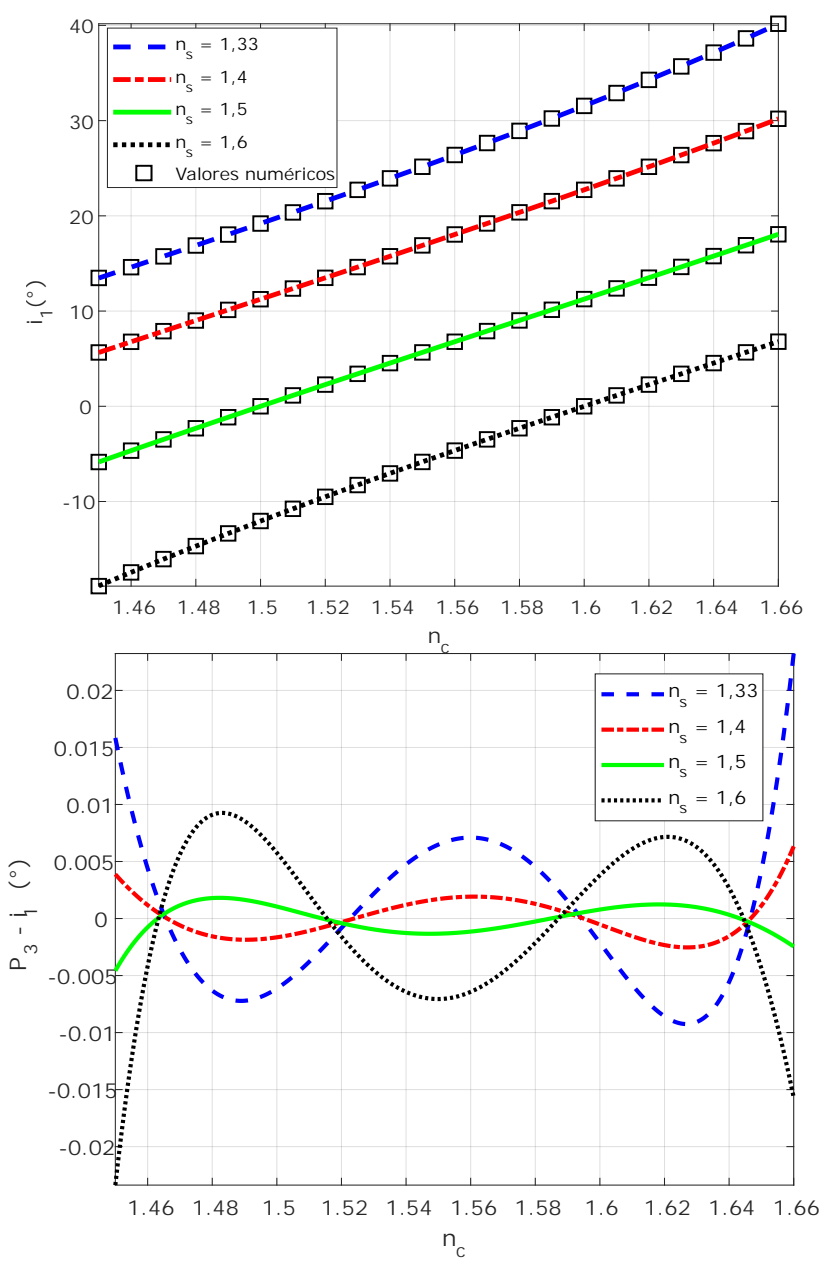

Figura 4: (Panel superior) Ángulo de incidencia calculado numéricamente (símbolos) y ajuste polinómico $P_{3}$ de orden 3 (líneas), en función del índice de refracción de la celda, para distintos valores de índices de refracción de la muestra. (Panel inferior) Error en la aproximación polinómica en función del material de la celda.
Estas curvas permiten determinar, a partir del rango de índices de refracción de las muestras, el material más conveniente a emplear en la construcción de la celda.

Para simplificar el cálculo, proponemos un ajuste polinómico de orden 3 que permite una buena aproximación (Ec. 8).

$$
P_{3}\left(n_{c}\right)=a n_{c}^{3}+b n_{c}^{2}+c n_{c}+d
$$

donde $a, b, c$ y $d$ son los coeficientes de ajuste, dados en la Tabla I.

Tabla I: Coeficientes del polinomio de ajuste $P_{3}$ para el ángulo de incidencia en función del índice de refracción del material de la celda.

\begin{tabular}{|c|c|c|c|c|}
\hline$n_{s}$ & 1,33 & 1,4 & 1,5 & 1,6 \\
\hline$a\left(^{\circ}\right)$ & 309,3 & 184,1 & 166,0 & 291,1 \\
\hline$b\left(^{\circ}\right)$ & $-1348,4$ & $-818,0$ & $-784,7$ & $-1430,4$ \\
\hline$c\left(^{\circ}\right)$ & 2073,4 & 1323,3 & 1348,3 & 2455,6 \\
\hline$d\left(^{\circ}\right)$ & $-1101,0$ & $-754,5$ & $-817,0$ & $-1459,7$ \\
\hline
\end{tabular}

En la Fig. 4 (Panel superior), se muestran también las aproximaciones polinómicas resultado del ajuste por cuadrados mínimos. A partir de las curvas representadas en este gráfico, se ve que, para un dado índice de la muestra, el ángulo de incidencia, necesario para salida normal, crece con el índice de refracción de la celda. A modo de ejemplo, para el caso de agua destilada, de índice de refracción 1,33, y una celda construida con prismas de índice 1,49, el ángulo de incidencia para salida normal es de $18^{\circ}$. Por otro lado, es claro que cuando el índice de refracción de la muestra es igual al del material de la celda, el haz luminoso no se desvía, lo que corresponde a incidencia normal en la cara frontal.

El error en la aproximación polinómica en función del material de la celda se muestra en la Fig. 4 (Panel inferior), para distintos índices de refracción de la muestra. En muchas aplicaciones, es conveniente minimizar el error introducido por la aproximación polinómica. A partir de la gráfica es posible entonces elegir el valor de $n_{c}$ más adecuado para el rango previsto de índices de refracción a medir.

Para dar una idea de la sensibilidad ante cambios en los parámetros de diseño, cabe destacar que, para una variación del índice de refracción del material de la celda de $10^{-5}$, la variación del ángulo de incidencia necesario para salida normal es del orden de 4".

Una vez elegido el material de los prismas, es necesario determinar la relación entre el ángulo de incidencia en la cara frontal del instrumento $\left(i_{1}\right)$ en función del índice de refracción de la muestra $\left(n_{3}\right)$, para salida normal. En la Fig. 5 , se presentan los resultados para tres valores de $n_{c}$. El menor corresponde a polimetilmetacrilato (PMMA), y los otros dos son valores típicos para vidrios ópticos tipo Crown y Flint, respectivamente. En la figura también se incluye la aproximación polinómica de tercer orden por cuadrados mínimos:

$$
Q_{3}\left(n_{s}\right)=g n_{s}^{3}+h n_{s}^{2}+j n_{s}+k
$$

donde $g, h, j$ y $k$ son los coeficientes de ajuste, cuyos valores se dan en la Tabla II. 

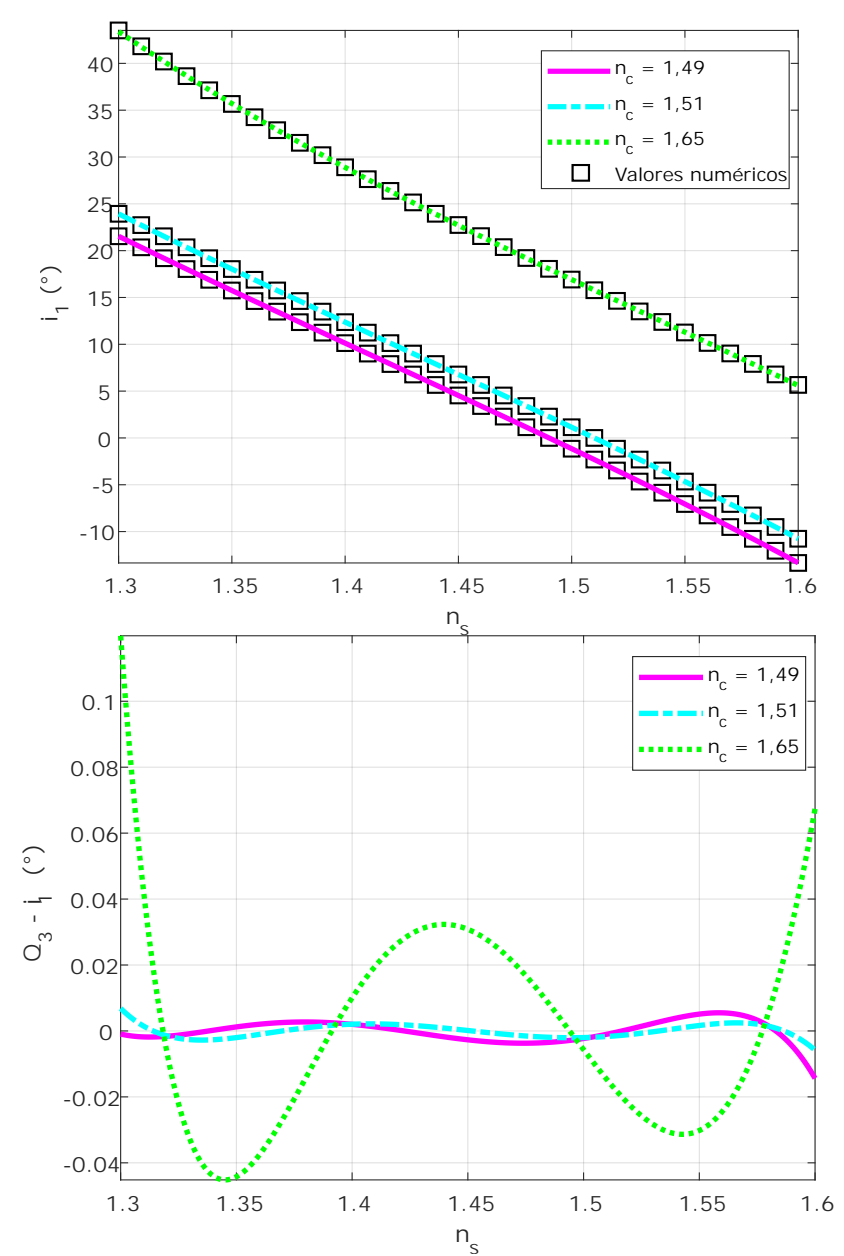

Figura 5: (Panel superior) Ángulo de incidencia calculado numéricamente (símbolos) y ajuste polinómico $Q_{3}$ de orden 3 (líneas), en función del índice de refracción de la muestra. (Panel inferior) Error en la aproximación polinómica en función del índice de refracción de la muestra.

Tabla II: Coeficientes del polinomio de ajuste $Q_{3}$ del ángulo de incidencia en función del índice de refracción de la muestra.

\begin{tabular}{|c|c|c|c|}
\hline$n_{c}$ & 1,49 & 1,51 & 1,65 \\
\hline$g\left(^{\circ}\right)$ & $-178,2$ & $-172,8$ & $-317,8$ \\
\hline$h\left(^{\circ}\right)$ & 755,2 & 743,3 & 1463,2 \\
\hline$j\left(^{\circ}\right)$ & $-1179,1$ & $-1177,2$ & $-2357,3$ \\
\hline$k\left(^{\circ}\right)$ & 669,3 & 677,9 & 1333,4 \\
\hline
\end{tabular}

En líneas generales, puede afirmarse que el ajuste es bueno, como surge de las curvas del error residual representadas en la Fig. 5 (Panel inferior).

En las aplicaciones de refractometría interesa determinar el índice de refracción de la muestra en función del ángulo de incidencia (Fig. 6). Proponemos, análogamente a los casos anteriores, un ajuste polinómico de orden 3 que permite una buena aproximación (Ec. 10).

$$
T_{3}\left(i_{1}\right)=l i_{1}^{3}+m i_{1}^{2}+u i_{1}+v
$$

donde $l, m, u$ y $v$ son los coeficientes de ajuste. Los mismos se dan en la Tabla III.

El error residual del ajuste se muestra en la Fig. 6 (Panel inferior). Cabe destacar que, para un dado valor de $n_{c}$,
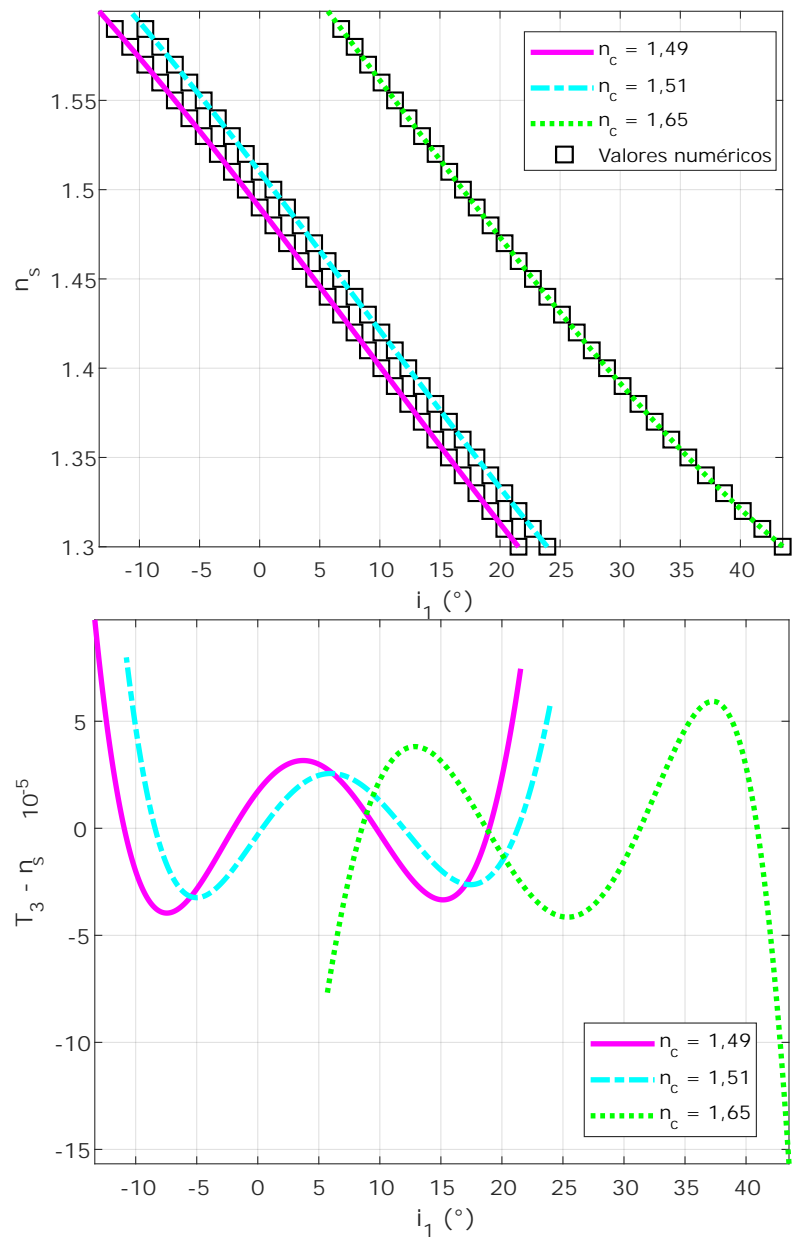

Figura 6: Índice de refracción de la muestra calculado numéricamente (símbolos) y ajuste polinómico $T_{3}$ de orden 3 (líneas), en función del ángulo de incidencia, para tres valores de índice de refracción del material de la celda (arriba). Error en la aproximación polinómica en función del ángulo de incidencia (abajo).

Tabla III: Coeficientes de ajuste polinómico $T_{3}$ del índice de refracción de la muestra en función del ángulo de incidencia.

\begin{tabular}{|c|c|c|c|}
\hline$n_{c}$ & 1,49 & 1,51 & 1,65 \\
\hline$l\left(1 /^{03}\right)$ & $-0,0425$ & $-0,0516$ & 0,0956 \\
\hline$m\left(1 /^{02}\right)$ & 0,1102 & 0,1086 & $-0,1257$ \\
\hline$u\left(1 /^{\circ}\right)$ & 0,2258 & 0,2201 & 0,2704 \\
\hline$v$ & 0,6712 & 0,6623 & 0,5968 \\
\hline
\end{tabular}

mayores valores de $i_{1}$ se corresponden con menores índices de refracción de la muestra $n_{s}$. Esto puede verse fácilmente a partir del análisis de las expresiones completas para las celdas en cuña [9].

Analizamos también la sensibilidad en aplicaciones de medición. Para esto, graficamos la derivada del índice de refracción de la muestra respecto al ángulo de incidencia, tanto en función del índice de refracción de la muestra (Fig. 7), como en función del índice de refracción de la celda (Fig. 8). De esta manera es posible estimar la incerteza en la determinación del valor de índice de refracción de la muestra. Además, a partir de la gráfica de la Fig. 8 es posible elegir el valor de $n_{c}$ para linealizar la dependencia de $i_{1}$ con $n_{s}$, eligiendo adecuadamente el material de los 


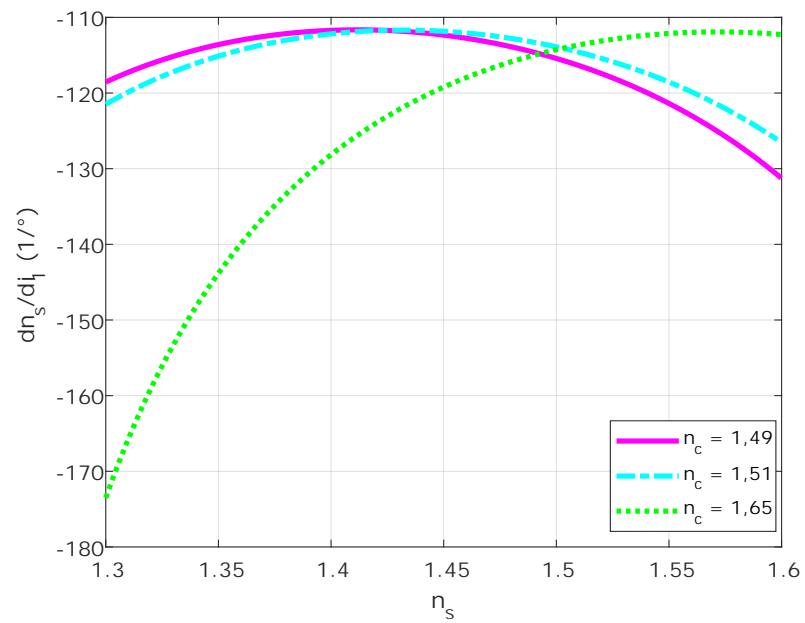

Figura 7: Derivada del índice de refracción de la muestra respecto al ángulo de incidencia, en función del índice de refracción de la muestra.

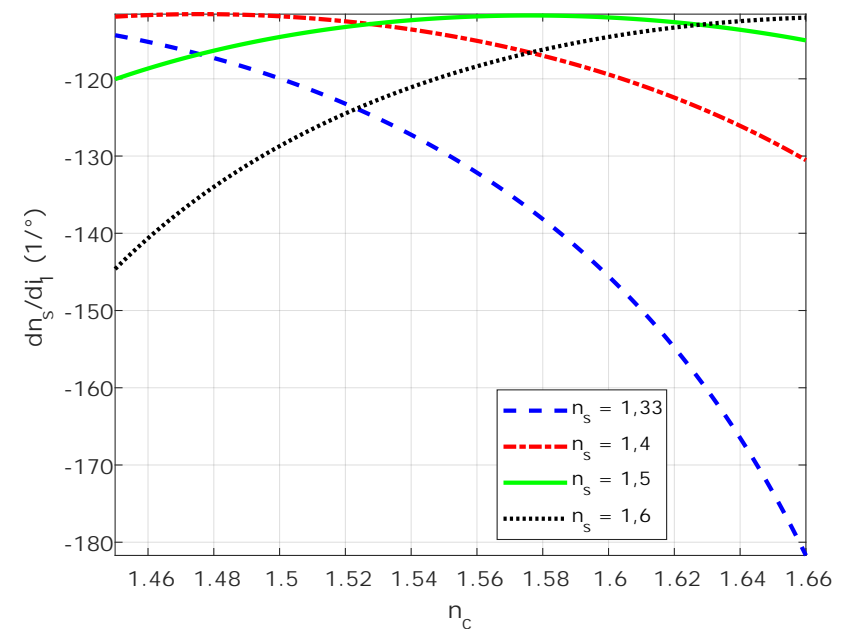

Figura 8: Derivada del índice de refracción de la muestra respecto al ángulo de incidencia, en función del índice de refracción de la celda.

prismas de modo de minimizar la variación de $\mathrm{d} n_{s} / \mathrm{d} i_{1}$ en el rango de índices de refracción de las muestras a medir. Esto es particularmente útil para aplicaciones de control de procesos.

Para validar este método, construimos una celda en acrílico (PMMA) con $n_{c}=n_{2}=n_{4}=1,49$ [11]. Elegimos trabajar con $\omega_{1}$ y $\omega_{3}$ iguales $\left(45^{\circ}\right)$ y $\omega_{2}$ recto $\left(90^{\circ}\right)$. En la cavidad se coloca el líquido cuyo índice de refracción se desea medir. Se hace incidir un haz de luz en un plano horizontal, ajustando el ángulo de incidencia en la cara frontal, $i_{1}$, mediante un espejo montado sobre un goniométro de precisión Newport UTR80A, que permite medir hasta el minuto de arco. El ángulo de incidencia se ajusta de forma tal que salga perpendicular a la cara posterior de la celda, $i_{3}^{\prime}=0$. En esas condiciones, a partir del ángulo de incidencia $i_{1}$ se puede determinar entonces el índice de refracción del líquido bajo análisis (ver Fig. 9). Este esquema experimental corresponde a una variante del esquema de Hughes [9], en el que trabaja en incidencia normal y rota el sistema de detección. Colocando como muestra agua destilada, de $n_{3}=1,33$, utilizando como fuente un láser de $\operatorname{HeNe}(\lambda=632,8 \mathrm{~nm})$ Melles Griot de $10 \mathrm{~mW}$, el ángulo de incidencia medido con el goniómetro fue $i_{1}=(18,00 \pm 0,33)^{\circ}$, en buen acuerdo con lo calculado en forma numérica, que es de $18^{\circ}$. Cabe destacar que el diseño de esta celda se destinará a mediciones de propiedades de soluciones acuosas de líquidos orgánicos, como parte de un trabajo actualmente en curso. En este trabajo elegimos mecanizar los prismas con catetos de $32 \mathrm{~mm}$. Ese valor es un buen compromiso entre facilidad de mecanizado y volumen de muestra.

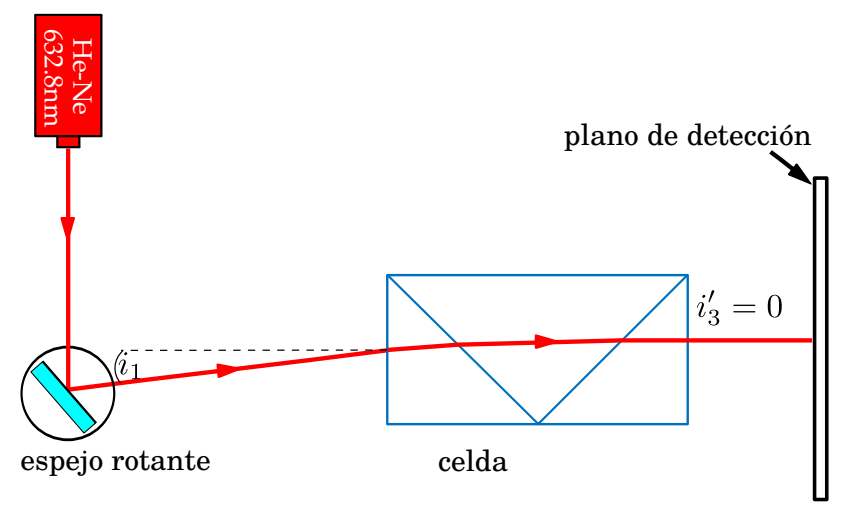

Figura 9: Esquema experimental utilizado para validar el método propuesto.

\section{CONCLUSIÓN}

En este trabajo presentamos los criterios de selección de materiales y parámetros de diseño de celdas en cuña para refractometría en el rango visible. Realizamos el cálculo numérico y el trazado de rayos, determinando el ángulo de incidencia para salida normal. De esta manera, conocida la geometría y el material de la celda, y medido el ángulo de incidencia, se determina el índice de refracción de la muestra. También se obtienen los puntos de incidencia y salida del haz. De esta manera es posible seleccionar el material de los prismas que conforman la celda, de acuerdo a las limitaciones geométricas y las propiedades de la muestra.

Presentamos ajustes polinómicos de orden 3 de los resultados numéricos, con sus correspondientes errores. Esto permite, por una parte, simplificar el análisis de los diversos parámetros de diseño de la celda y, por otra parte, obtener una expresión explícita del índice de refracción de la muestra en función del ángulo de deflexión. Los errores en los ajustes son pequeños, y disminuyen para menores valores del índice de refracción de la celda. Incluimos también el análisis de la sensibilidad para la determinación del índice de refracción de la muestra respecto del ángulo de incidencia en la cara frontal de la celda.

\section{AgRADECIMIENTOS}

Este trabajo fue financiado por los subsidios UBACyT 20020160100052BA y UBACYT 20020170200232BA de la Universidad de Buenos Aires y el subsidio PICT 2016-2204 de la ANPCyT.

\section{REFERENCIAS}

[1] S. D. Romano and P. A. Sorichetti, Dielectric relaxation spectroscopy in biodiesel production and characterization, 1st ed. Springer Verlag, 2011. 
Revista elektron, Vol. 4, No. 1, pp. 8-13 (2020)

[2] B. F. Liptak, Instrument engineer's handbook: process measurement and analysis, 4th ed. CRC Press, 2003.

[3] A. Chianese and H. J. Kramer, Industrial crystallization process monitoring and control. Weinheim: Wiley-VCH Verlag and Co., 2012.

[4] A. W. S. Tarrant, Intrumentation Reference Book, Cap. 28, 4th ed. Elsevier, 2010.

[5] H. Hattori, H. Kakui, H. Kurniawan, and K. Kagawa, "Liquid refractometry by the rainbow method," Applied optics, vol. 37, no. 19, pp. 4123-4129, 1998.

[6] B. M. Pixton and J. E. Greivenkamp, "Automated measurement of the refractive index of fluids," Applied Optics 47, p. 1504, 2008.

[7] U. Petzold, R. U., Jedamzika, P. Hartmanna, and R. S., "V-block refractometer for monitoring the production of optical glasses," Proc. of SPIE Vol. 9628, p. 962811, 2015.

[8] M. Le Menn, "Calibration and temperature correction of a v-block refractometer," Meas. Sci. Technol. 29, p. 037001, 2018.

[9] J. V. Hughes, "A new precision refractometer," J. of Sci. Instrum. 18, p. $234,1941$.

[10] M. S. Millán, J. Escofet, and E. Pérez, Óptica geométrica. Ariel, 2004.

[11] E. V. Oreglia, P. M. E. Vázquez, C. L. Matteo, P. A. Sorichetti, F. E. Veiras, and L. Ciocci Brazzano, "Medición simultánea de velocidad del sonido e índice de refracción en líquidos." 10ma Reunión Ibero Americana de Óptica y 13vo Encuentro Latinoamericano de Óptica, Lasers y sus aplicaciones (RIAO-OPTILAS), 2019. 